\title{
Paradise revealed II: Top science, sunny beach, tropical fruits and caipirinhas....
}

\author{
GP Amarante-Mendes ${ }^{\star, 1,2}$, S Adjemian ${ }^{1}$, BP Mello ${ }^{1}$, ED Hottz ${ }^{3}$ and PE Marques ${ }^{4}$
}

Cell Death and Differentiation (2015) 22, 1231-1233; doi:10.1038/cdd.2014.242; published online 30 January 2015

Cell Death Signaling in Cancer and the Immune System (Keystone Symposia on Molecular and Cellular Biology), Guarujá, São Paulo, Brazil, 28 October-2 November 2014

Some people say that lightning never strikes twice in the same place. Gratefully, sunbeam does! In February 2010, Brazilian scientists organized an outstanding multidisciplinary meeting at a beach resort in Angra dos Reis, Rio de Janeiro, Brazil, that brought together leaders of the cell death, tumor biology and immunology fields. Along with couple of hundred participants, speakers shared their latest work, experience and enthusiasm in an excited atmosphere rocked by the sound of waves. ${ }^{1}$ Keystone Symposia on Molecular and Cellular Biology and Fundação de Amparo à Pesquisa do Estado de São Paulo (FAPESP), the São Paulo State Research Foundation, decided to surf this idea and worked together to implement the first Keystone meeting in the State of São Paulo-the second organized in Brazil. This time the venue was the delightful Enseada beach, in Guarujá, a coastal paradisiacal town located in the south of the State of São Paulo. The sophisticated and yet casual hotel, the amazing fine sand beach, the fresh breeze, the tasty fruits and coconut water and, of course, the famous Brazilian caipirinhas provided an incredibly relaxed and friendly atmosphere where top scientists were overwhelmed by the quality and depth of students' interrogations.

Organized by Gustavo Amarante-Mendes, Douglas Green and Kim Newton, the event started with a pre-meeting course on Cell Death modalities. The course brought together 60 PhD students or postdocs fully supported by FAPESP, half coming from Brazil and half from abroad, creating a dynamic and highly interactive environment that lasted throughout the meeting. Vishva Dixit (Genentech, Inc., USA) presented the 'Keynote Address' on inflammasome structure and signaling events, describing the activation of non-canonical inflammasome by cytosolic LPS and its role in septic shock. In line with this, Petr Broz (University of Basel, Switzerland) demonstrated that guanylate-binding proteins (GBPs) targeted the bacteria and promoted disruption of bacteria-containing vacuoles, allowing cytosolic sensing of LPS and caspase-11 activation. Guy Salvesen (Sanford-Burnham Medical Research Institute, USA) introduced us to novel specific probes highly specific for each caspase active site, enabling unambiguous discrimination of caspase activation. Mohanish Deshmukh (University of North Carolina at Chapel Hill, USA) demonstrated that cytosolic cytochrome $c$ is not able to engage in the apoptosome formation in neurons because of very low levels of Apaf-1 in these cells, and that free cytosolic cytochrome $c$ is ubiquitinated and degraded by the proteasome. Marion MacFarlane (MRC Toxicologic Unit, UK), demonstrated that caspase-8 and c-FLIP were able to form chains, and that the respective ratio of caspase-8/c-FLIP $\mathrm{S} / \mathrm{L}$ determines whether the cell would engage the apoptotic pathway or rather continue to live. Necroptotic pathways were extensively discussed by Peter Vandenabeele (VIB, Ghent University, Belgium), Francis Chan (University of Massachussets Medical School, USA) and Kim Newton (Genentech, Inc., USA). Peter Vandenabeele described that RIPK1 protects intestinal epithelial cells (IECs) from apoptosis. RIPK1 $1^{\mathrm{IEC}-\mathrm{KO}}$ mice presented increased apoptosis, barrier disruption, bacterial translocation and inflammatory infiltrates, resulting in significant weight loss and lethality. Importantly, antibiotic treatment of RIPK1 ${ }^{\mathrm{IEC}-\mathrm{KO}}$ mice decreased the inflammation and improved survival. Francis Chan revealed that, in dendritic cells, RIPK3 is necessary for TLR4-mediated activation of caspases-8 (requirement for FADD and RIPK1) and -1 (requirement for ROS) and IL-1 $\beta$ processing, and that RIPK3-mediated maturation of IL- $1 \beta$ is critical to injury-induced tissue repair. Kim Newton described that necroptosis-related inflammation is dependent on RIPK1 and RIPK3, but not on MLKL. Douglas Green (St Jude Children's Research Hospital, USA) delivered a captivating lecture in which he approached the question of non-genetic control of cell survival. He presented new results

${ }^{1}$ Departamento de Imunologia, Instituto de Ciências Biomédicas, Universidade de São Paulo, São Paulo, Brazil; ${ }^{2}$ Instituto de Investigação em Imunologia, Instituto Nacional de Ciência e Tecnologia,São Paulo, Brazil; ${ }^{3}$ Laboratório de Imunofarmacologia, Instituto Oswaldo Cruz, Fundação Oswaldo Cruz, Rio de Janeiro, Brazil and ${ }^{4}$ Departamento de Morfologia, Instituto de Ciências Biológicas, Universidade Federal de Minas Gerais, Belo Horizonte, Brazil

*Corresponding author: GP Amarante-Mendes, Departamento de Imunologia, Instituto de Ciências Biomédicas, Universidade de São Paulo, Av. Professor Lineu Prestes, 1730, Cidade Universitária—05508-900, São Paulo, SP 05508-000, Brazil. Tel: +55 113091 7362; Fax: +55 113091 7224; E-mail: gpam@usp.br 
obtained by single-cell mass spectrometry to investigate the cell population that consistently survives TRAIL-induced apoptosis.

\section{Cell Death and Host Defense}

Ruslan Medzhitov (HHMI/Yale University School of Medicine, USA) talked about local tissue-derived signals as controllers of functional polarization of resident macrophages and whether functional heterogeneity is the result of irreversible lineagespecific differentiation or of continuous but reversible induction of diverse functional programs. Karina Bortoluci (Federal University of São Paulo, Brazil) presented novel evidences about the interactions among different inflammasomes. She showed that both NLRP3 and NLRC4 are required for IL-1 $\beta$ secretion in response to bacterial flagellin and that this response also requires lysosomal cathepsins. Moreover, she demonstrated that flagellin-induced IL-1 $\alpha$ and IL- $1 \beta$ by dendritic cells mediate the induction of Th1-immune responses; a possible mechanism involved in the adjuvant properties of this protein. Igor Brodsky (University of Pennsylvania, USA) dissected the mechanisms for Yersinia protein YopJ-induced cell death. YopJ-induced caspase-1 activation required RIPK1, FADD and caspase-8 catalytic activity. RIPK3 was not required for caspase-1-mediated YopJinduced cell death, but in the absence of caspase-8, RIPK3 triggered necrosis. These cell death pathways had important roles in bacterial restriction in mice. Thirumala-Devi Kanneganti (St Jude Childrens' Research Hospital, USA) showed that RIP-1 or IL-1a knockout totally rescued SHP1 ${ }^{-/-}$ mice from skin inflammation. On the other hand, osteomyelitislike autoinflammatory bone disease developed in PSTPIP2deficient mice depends on IL-1R and IL-1 $\beta$, but not on IL-1a expression. Interestingly, inflammation in these models was driven by microbiota, and Provetella removal through high fatty diet rescued PSTPIP2 ${ }^{-/-}$and $\mathrm{SHP}^{-1-}$ mice from bone and skin inflammatory disease, respectively. Janelle Ayres (The Salk Institute, USA) argued that Enterobacteriales is associated with protection against wasting syndrome. Horizontal transferring or direct administration of Enterobacteriales protected mice from changes in muscle, lean and white adipose tissue metabolism, and wasting syndrome mortality, without affecting pathogen levels or systemic and localized inflammation. Gustavo Amarante-Mendes (University of São Paulo, Brazil) showed that galectin-1 is part of the cytotoxic granule machinery and has a role in the in vivo CD8 T-cell elimination of antigen-specific targets, possibly by regulating FAS/FASL interactions. The mechanisms involved in the IL-1 secretion are a matter of intense debate in the literature. Taking advantage of a single-cell assay, Seamus Martin (Trinity College Dublin, Ireland) demonstrated that IL- $1 \beta$ is released only by dead cells in response to several inflammasome agonists, challenging the idea of the existence of active mechanisms for secretion of this cytokine. Andrew Oberst (University of Washington, USA) used a system in which apoptotic or necroptotic effector enzymes are activated indirectly by a non-toxic molecule to find out that necroptotic cells are uniquely inflammatory and immunogenic.

\section{Consequences of Cell Death}

The multiple impacts of cell death in health and disease were thoroughly discussed during the meeting. Shigekazu Nagata (Kyoto University, Japan) nailed down the mechanisms responsible for phosphatidylserine (PtdSer) exposure during apoptosis. He showed that effector caspases proteolitically activate the scramblase Xk-related family protein 8 (XKR8) and inactivate the flippase adenosine triphosphatase type-11C (ATP11C). Interestingly, both events seem to be necessary for PtdSer exposure during apoptosis. Studying the salivary gland of Drosophila, Eric Baehrecke (University of Massachusetts Medical School, USA) showed that autophagy is necessary for proper tissue development and function, measured by histology and salivary gland protein secretion, respectively. Will Wood (University of Bristol, UK) demonstrated that, in the fly embryo, in vivo laser wounding triggers a rapid calcium wave in the epithelium, which activates the fly NADPH oxidase (DUOX) and promotes hydrogen peroxide production, causing a fierce migration of hemocytes to the injury site.

\section{Cell Death in Cancer}

Andreas Strasser (Walter and Eliza Hall Institute of Medical Research, Australia) used a $p 21^{-1-}$ puma $^{-1-}$ noxa ${ }^{-/-}$tripleknockout mice to find that transcriptional induction of these genes is not essential for p53's ability to prevent spontaneous tumor development. David Andrews (University of Toronto, Canada) discussed about new perspectives on the mechanisms by which $\mathrm{Bcl}-2$ family proteins interact with membranes and with each other, that have been revealed by their fluorescence measurements of binding affinities in vitro and in live cells (quantitative FLIM-FRET). Gerry Melino (University of Rome 'Tor Vergata', Italy) presented evidences that miR-203 is a key molecule controlling the p63-dependent proliferative potential and morphogenesis of epithelial (keratinocytes) and glandular (prostate cancer) tissues by regulating the level of DeltaNp63 expression. Gerard Evan (University of Cambridge, UK) revealed that the integration of mechanisms such as metabolic dysfunction, collapse of tumor vasculature, differentiation and ablation of immune blockade due to downregulation of ligands that repress $\mathrm{T}$ cells, is the way that Myc inhibition selectively kills cancer cells. Karen Vousden (Beatson Institute for Cancer Research, UK) showed that p53 helps to support the adaptation of cancer cells to nutrient depletion (such as glucose, glycine or serine starvation) by inducing the transcription of TIGAR, and that many transformed cells are highly dependent on the uptake of exogenous serine. By perturbing mitochondria with $\mathrm{BH} 3$ peptides to measure how close a cell is to the threshold of apoptosis (priming), Tony Letai (Dana-Farber Cancer Institute, USA) found that the more sensitive a mitochondrion is to $\mathrm{BH} 3$ peptides, the more primed the cell is to death. Also, he demonstrated that priming predicts clinical sensitivity to chemotherapy and that this $\mathrm{BH} 3$ profiling can be used to detect dependence on BCL-2 and BCL-XL for survival, predicting cytotoxic response to small molecule antagonists, such as ABT-199 
and ABT-263. Simone Fulda (University Hospital Frankfurt, Germany) and David Huang (Walter and Eliza Hall Institute of Medical Research, Australia) captained that evasion of apoptosis is still a major problem for anticancer therapies. Fulda focused on aberrant expression of inhibitor of apoptosis proteins in tumors, which can be therapeutically targeted by a SMAC mimetics, whereas Huang concentrated his attention to the BCL-2 family and the use of $\mathrm{BH} 3$ mimetics, particularly ABT-199. Both presented convincing clinical evidences that these are promising tools in our long lasting fight against cancer. Closing remarks were provided at the Keynote Lecture presented by Scott Lowe (HHMI/Memorial Sloan-Kettering Cancer Center, USA).
Dr Lowe presented their new mouse cancer models based on the genetic manipulation of stem and progenitor cells ex vivo followed by transplantation of the altered cells into the appropriate organ of syngeneic recipient mice. Also, he showed work on developing methods for using miR-based RNA interference to suppress gene function in vivo.

We apologize as owing to space restrictions we were not able to discuss in full these talks or many other interesting oral and poster presentations.

1. Amarante-Mendes GP, Bortoluci KR, Bozza PT, Chammas R, Viola JP. Paradise revealed: first-class science rocked by the sound of the waves. Cell Death Differ 2010; 17: 1368-1372. 